\title{
GAIA Level 1 Neonatal Respiratory Distress
}

National Cancer Institute

\section{Source}

National Cancer Institute. GAIA Level 1 Neonatal Respiratory Distress. NCI Thesaurus. Code C128768.

GAIA Level 1 Neonatal Respiratory Distress is defined by four criteria: first, the newborn must be between 0 and 28 days of life; second, the presence of any one of the following three symptoms that indicate an abnormal respiratory rate: a) Tachypnea (respiratory rate of 60 or more breaths per minute); b) Bradypnea (respiratory rate of less than 30 breaths per minute); c) Apnea [cessation of respiratory effort (no breaths) for at least 20 seconds]; third, one of the following five clinical symptoms that indicate labored breathing: a) Nasal flaring (dilatation of alae nasi); b) Noisy respirations in the form of expiratory grunting, stridor, or wheeze; c) Retractions or increased chest indrawings on inspiration (subcostal, intercostal, sternal, suprasternal notch); d) Central cyanosis (whole body, including lips and tongue) on room air; e) Low Apgar score (less than 7 points) at 10 minutes, with respiration score less than 2; fourth, examination and documentation by qualified, trained health care providers who are appropriate for the clinical setting. 